\title{
A Unique Case Report of Polyneuropathy with Lumbar Radiculopathy
}

\author{
Anjali S. Bais', Simran A. Mishra', Palak P. Darda , Waqar M. Naqvi \\ ${ }^{1}$ Intern, Ravi Nair Physiotherapy College, Datta Meghe Institute of \\ Medical Sciences, Wardha, Maharashtra, India. \\ Corresponding author email: anjali.bais.9097@gmail.com
}

\begin{abstract}
Disability is obvious when lumbar radiculopathy are diagnosed with polyneuropathy. About $27 \%$ lumbar radiculopathy cases are such complex cases. The key treatment modality includes Physiotherapy rehabilitation. Studies have been reported on Physiotherapy rehabilitation to be effective in 50\% patients, without any surgery. This case report attempted to reflect detailed phase wise rehabilitation and treatment protocol for neuropathy. Patient's chief complaints were low back pain radiating to both lower limbs and difficulty in walking. He was unable to performroutine activities smoothly and independently. Physical examination revealed reduced lumbar lordosis, absent sensation and hypoesthesia. Also diminished and absent deep tendon reflexes were noted with reduced strength, SLR 50 bilaterally. motor polyneuropathy with lumbar radiculopathy. Lumbar radiculopathy was confirmed by MRI of lumbar spine. Polyneuropathy was confirmed by Nerve Conduction Velocity. Lab. Conformation of decreased serum copper in patients body reflecting the cause of sensory motor polyneuropathy. Physical therapy intervention included range of motion exercises, strengthening exercises, sensory retraining, balance and co-ordination training strategies, and Electrical Muscle stimulation. A significant effect of the 20 weeks of intensive physiotherapeutic interventionreflected on the co-ordination, balance, gait, ADLs, muscle strength, muscle re-education, and functional independence of the patient.
\end{abstract}

KEY WORDS: LATERAL EPICONDYLITIS, TENNIS ELBOW, PHYSIOTHERAPY, HOME EXERCISE PROGRAMME, TENDINOPATHY, MILLS MANIPULATION.

\section{INTRODUCTION}

Polyneuropathy is syndrome rather than specific disease (Dyck, 1981). Though there are multiple causes, detailed review of history, complete clinical evaluation, electrodiagnostic studies and laboratory testing's can reveal etiology in almost $76 \%$ of patients. In young adults prevalence is approx. 2-4\% (Martyn, 1997; Peripheral, 2020). Lumbar radiculopathy refers to pathological process involving lumbar nerve roots causing radicular symptoms into lower extremities (Della, 2015, Physical, 2020). Nerve root pathology arises primarily from direct

Biosc Biotech Res Comm P-ISSN: 0974-6455 E-ISSN: 2321-4007

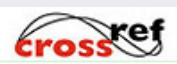

Identifiers and Pagination

Year: 2021 Vol: 14 No (6) Special Issue

Pages: 87-94

This is an open access article under Creative

Commons License Attribn 4.0 Intl (CC-BY).

DOI: DOI: $h t t p: / / d x . d o i . o r g / 10.21786 / b b r c / 14.6 .21$ compression irrespective of etiology. Prevalence of lumbar radiculopathy is $2.2-8 \%$.

Approx. 27\% of patients with lumbar radiculopathy are diagnosed with polyneuropathy and when both occurs together complex situation occurs that is capable of causing disability (clinical 2019). Physiotherapy rehab for disc disease/ lumbar radiculopathy have long been addressed in literatures, found effective and suggests possible recovery in 50\% patients without surgery (Berry et al., 2020; Clinical, 2020, Zhang, et al, 2019). However detailed phase wise rehab for neuropathy hasn't been specified yet and despite availability of various diagnostic modalities, most of times polyneuropathy are misdiagnosed or late diagnosed (Allen , 2020; Peroneal , 2020; Singh, 2015). This warrants new studies to provide detailed and precised treatment protocol.

In this case, occurrence of lumbar radiculopathy and MRI impression of degenerative changes in spine, initially diverts attention from neuropathy however clinical 
evaluation, electro diagnostic studies and lab testing's confirmed polyneuropathy.

Patient informlation: 35 year old female, farm worker visited Physiotherapy OPD, on 30 Jan 2020, walking with the support of her mother in law. Relative described complaints, Low back Pain (LBP) radiating to both lower limbs since 2 months which was insidious on onset and gradually progressive, aggravates on walking, relieves with medication and varies between 4-7 on NPRS. After a month symptoms further progressed pain was 8 on NPRS this time associated with tingling and numbness in lower limbs accompanied by unsteady gait which further deteriorate and render patient unable to walk unaided or without support. She doesn't look well-nourished and has BMI.

$18.3 \mathrm{~kg} / \mathrm{m} 2$ (under-weight) there was no relevant past history or family history of neurological diseases.

Clinical Sindings: On admission, complete evaluation and assessment was done

a. Initial examination of mental status can't be done due to uncooperative attitude of patient, however on gross evaluation and with help of information provided by care giver patient seems to have behavioral problems and poor comprehension.

b. Sensory examination revealed absent bilateral sensation (touch, pain, temperature) in L5,S1,S2 and reduced in $\mathrm{L} 2, \mathrm{~L} 3, \mathrm{~L} 4$.

Table 1. Sensations and grades

\begin{tabular}{|l|c|c|}
\hline Dermatomes & $\begin{array}{c}\text { Grading } \\
\text { Left }\end{array}$ & Right \\
\hline L1 & 1 & 1 \\
\hline L2 & 1 & 1 \\
\hline L3 & 1 & 1 \\
\hline L4 & 1 & 1 \\
\hline L5 & 0 & 0 \\
\hline S1 & 0 & 0 \\
\hline S2 & 0 & 0 \\
\hline
\end{tabular}

c. Motor Assessment, complete evaluation of Range of Motion (ROM), joint play, reflexes, strength and soft tissue compliance was done.

\section{Reflexes}

Deep tendon reflexes :- Upper extremities were normal bilaterally. In Lower extremity knee reflex were normal (grade 2) and ankle reflex were absent (grade 0) bilaterally.

Superficial Plantar reflex was absent (mute babinski).

Muscle Strength: Strength of all muscle groups in upper extremity is normal, grade 5 on MRC and finger grip is $100 \%$.
Tone: Muscle tone is normal in all the muscle groups of upper as well as lower extremity

\section{d. Coordination assessment}

e. Gait

Evaluation can't be done as patient is unable to walk unsupported and having difficulty in maintaining balance, however on the basis of observation when patient came to department, seems to be walking with ataxic gait with wide base of support, irregular foot placement, sway (swinging sideways) and audible slapping of foot.

Table 2. Range Of Motion

Right side Left Side

\begin{tabular}{|l|l|l|l|l|}
\hline Active & Passive & Active & Passive \\
\hline
\end{tabular}

1.Hip Jt.

Flexion

Extension

Abduction

Adduction

\begin{tabular}{|l|l|}
\hline $0-85$ & $0-90$ \\
\hline $0-25$ & $0-30$ \\
\hline $0-30$ & $0-35$ \\
\hline $0-15$ & $0-20$ \\
\hline
\end{tabular}

\begin{tabular}{|l|l|}
\hline $0-90$ & $0-95$ \\
\hline $0-30$ & $0-30$ \\
\hline $0-40$ & $0-45$ \\
\hline $0-18$ & $0-20$ \\
\hline
\end{tabular}

2.Knee Jt.

Flexion

Extension

\begin{tabular}{|l|l|}
\hline $0-110$ & $0-115$ \\
\hline $110-0$ & $115-0$ \\
\hline
\end{tabular} \begin{tabular}{|l|l|}
\hline $0-125$ & $0-130$ \\
\hline $120-0$ & $130-0$ \\
\hline
\end{tabular}

3. Ankle Jt.

Plantarflexion

Dorsiflexion

\begin{tabular}{|l|l|}
\hline $0-30$ & $0-35$ \\
\hline $0-8$ & $0-12$ \\
\hline
\end{tabular}$\quad$\begin{tabular}{|l|l|}
\hline $0-15$ & $0-20$ \\
\hline NA & $0-10$ \\
\hline
\end{tabular}

Table 3. Muscle strength and grades

\begin{tabular}{|l|c|c|c|}
\hline Muscle Groups & $\begin{array}{c}\text { Gradings } \\
\text { Hip }\end{array}$ & Left & Right \\
\hline \multirow{7}{*}{ Flexors } & 5 & 5 \\
\hline Extensors & 5 & 5 \\
\hline Adductors & 5 & 5 \\
\cline { 2 - 4 } & Abductors & 5 & 5 \\
\hline Knee & & \\
\hline Flexors & 5 & 5 \\
\hline Extensors & 5 & 5 \\
\cline { 2 - 4 } & Plantaflexors & 2 & 2 \\
\cline { 2 - 4 } & Dorsiflexors & 2 & 2 \\
\hline
\end{tabular}

Diagnosis: Several diagnostic procedures were done and helped to verify the cause as well as the condition. 
A. Laboratory Investigation: Serum $\mathrm{Cu}$ and serum $\mathrm{Zn}$ ranges has been evaluated and patient was diagnosed with deficiency of both the trace elements in her body.

B. Electrophysiological Investigation- Both sensory and motor nerve conduction studies were done

Findings of NCV report revealed:

a. CMAP amplitude could not be elicited in B/L tibial,peroneal nerve.

b. F min latency could not be elicited in B/L tibial,peroneal nerve.

c. SNAP amplitude could not be elicited in $\mathrm{B} / \mathrm{L}$ sural nerve.

All the above values were within the normal limits for median and ulnar nerve.

Patient was diagnosed with sensory motor polyneuropathy in lower limb bilaterally.

Table 4. Coordination test and grades

\begin{tabular}{|l|c|c|}
\hline & Right side & Left side \\
\hline Non equilibrium tests & & \\
\hline Tapping(foot) & 3 & 3 \\
\hline Alternate heel to knee & 2 & 2 \\
\hline Alternate heel to toe & 2 & 3 \\
\hline Heel on shin & 1 & 2 \\
\hline Drawing a circle(foot) & 3 & 3 \\
\hline
\end{tabular}

\begin{tabular}{|l|c|}
\hline Equilibrium tests & \\
\hline SITTING & \\
\hline -in normal comfortable position & Good \\
\hline -weight shift in all directions & Fair \\
\hline -multidirectional functional reach & Fair \\
\hline STANDING & \\
\hline -on one foot & Poor \\
\hline -tandem standing & Poor \\
\hline -Rhombergs (eyes open to eyes close) & Absent \\
\hline -Sharpened Rhombergs (E0 to EC) & Absent \\
\hline
\end{tabular}

C. Radiological Investigation

1. MRI of Lumbar spine

The Findings of MRI report revealed:

2. MRI of Brain were done and report revealed chronic lacunar infarct in right lentiform nucleus

Diagnostic Challenges: Patient lives in rural area and is having lack of access to proper healthcare facilities and lack of awareness which led to severe progression of disease and late diagnosis. Patient had financial instability which became the barrier for proper diagnostic procedures as well as surgical interventions. Patient was not co-operative and were suffering from forgetfulness, behavior problems, poor comprehension which interfered with the diagnostic procedure.

Intervention: Patient underwent intensive physical therapy in order to prevent further risks and complications as well as to maintain and to increase muscle strength, to improve sensory awareness and functional ability of patient.
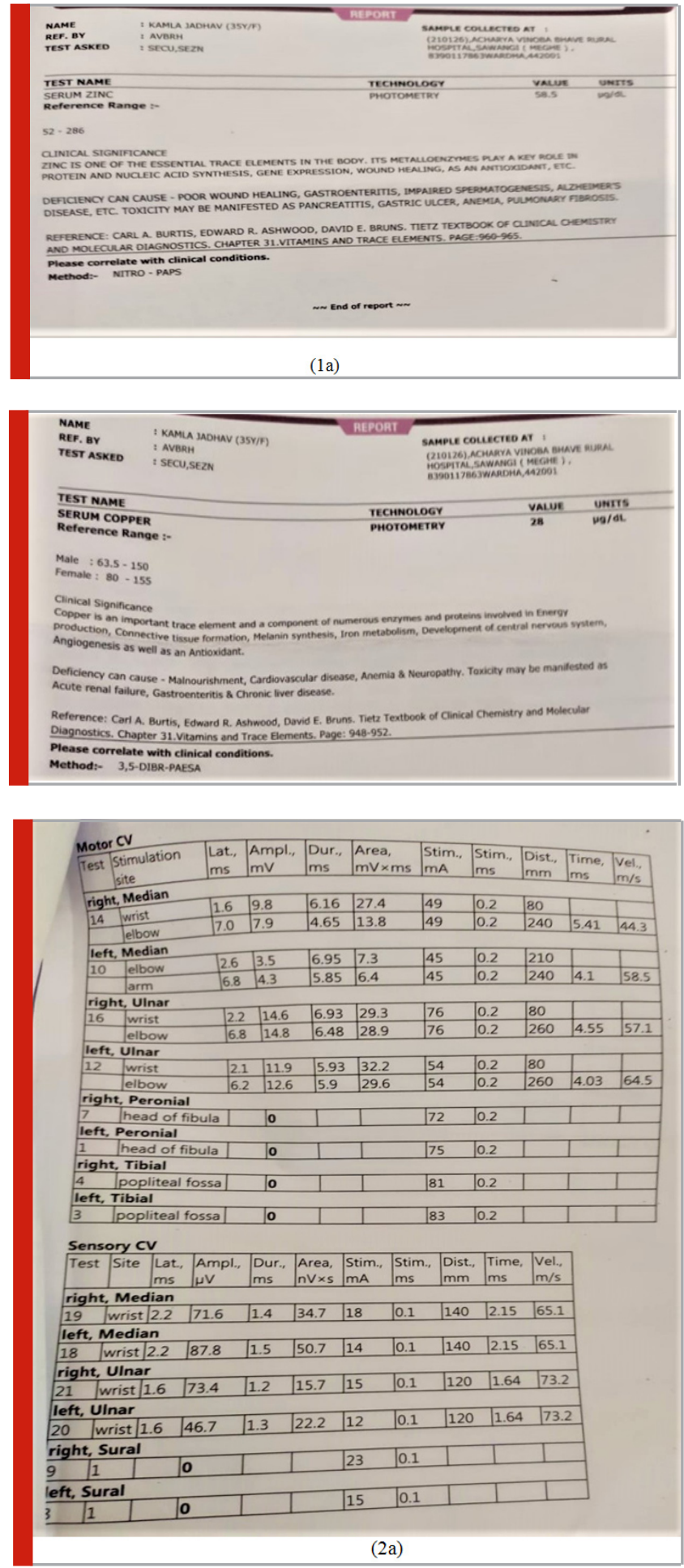
The 20 weeks rehabilitation protocol has been given which includes patient education, splinting and positioning, Range of motion (ROM) exercises, stretching and strengthening exercises, electrical stimulations, sensory retraining and motor retraining, balance and coordination exercises and functional exercises.

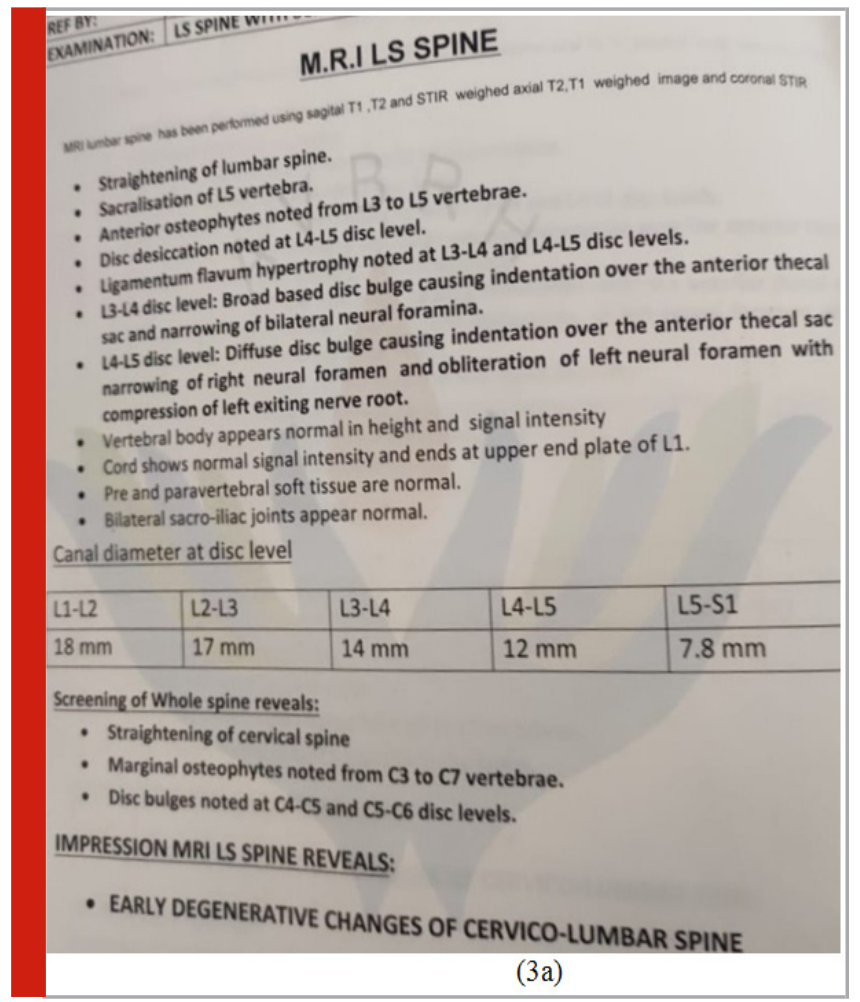

\section{Week 1 To Week 4:}

Thermotherapy: Prior to treatment, patient was given heating modality (hot pack) for 10-15 minutes.

ROM exercise- Active-assisted range of motion for hip flexors, extensors, abductors, adductors, internal and external rotators. Similarly, active-assisted ROM for knee flexors and extensors and passive ROM of ankle plantarflexors and dorsiflexors were given 2 times a day with 10 repetitions in order to prevent contractures, stiffness and deformity.

Bracing for lower back as well as foot drop were given to patient

1. Lumbar corset has been prescribed in order to prevent further mal alignment of spine and to give spine a proper stability. Patient used to wear it while performing any standing or sitting activities.

2. AFO has been prescribed to patient while extremity is at rest in order to allow passive dorsiflexion of ankle as well as reduce to reduce risk of contracture.

Stretching Exercises: Stretching of major group of muscle i.e Quadriceps and hamstrings as well as tendo-achilles has been given twice daily with 15 seconds hold. The stretches were of low intensity in order to provide effective elongation in dense connective tissue.
Electrical Stimulation- Electrical muscle stimulation was given to the affected muscles (current type-Intermittent Galvanic; Wavelength-Triangular; duration-15min) for effective muscle activation. It has been given to Tibialis Anterior, Peroneus longus, Extensor digitorum longus and Extensor hallucis longus.

Care of skin: patient also presented complaints of hypoesthesia down from L4 level. So, special attention was given to prevent breakdown or injury or excessive stretch to that area while performing any activities or giving any modalities.

\section{Week 4 To Week 12:}

ROM- Hip and Knee ROM has been given 2 times a day with progression of 15 repetition and patient started performing it actively, Ankle ROM was performed with the same dosage but required assistance.

Stretching duration was progressed from 15 to 30 seconds.

\section{Strengthening Exercises:}

1. Patient started performing Core strengthening exercises i.e Static abdominals, Static back, Pelvic Bridging with 10 seconds hold and 10 repetitions twice a day.

2. Static Quadriceps and Static Hamstring exercises were given to patient with 10 seconds hold and 10 repetition twice a day.

Electrical stimulation was changed to faradic stimulation with 30 to 60 contactions and 10 minutes of duration and patient performed or attempted to initiate the action of muscle voluntarily.

Sensory Training Strategies: Effective strategies has been given to patient in order to improve the sensations as patient also suffered from hypoesthesia and it also acts as a prerequisites for gait training as patient suffered from sensory ataxia

a. Application of vibratory massager was used in order to overcome the inability to sense vibration. That was then used over the unaffected area so that patient could know the difference.

b. Patient used to stand and walk on a different texture and uneven surface for 5-10 minutes

and patient performed or attempted to initiate the action of muscle voluntarily.

Sensory Training Strategies: Effective strategies has been given to patient in order to improve the sensations as patient also suffered from hypoesthesia and it also acts as a prerequisites for gait training as patient suffered from sensory ataxia

a. Application of vibratory massager was used in order to overcome the inability to sense vibration. That was 
then used over the unaffected area so that patient could know the difference.

b. Patient used to stand and walk on a different texture and uneven surface for 5-10 minutes and patient performed or attempted to initiate the action of muscle voluntarily.

Sensory Training Strategies: Effective strategies has been given to patient in order to improve the sensations as patient also suffered from hypoesthesia and it also acts as a prerequisites for gait training as patient suffered from sensory ataxia

a. Application of vibratory massager was used in order to overcome the inability to sense vibration. That was then used over the unaffected area so that patient could know the difference.

b. Patient used to stand and walk on a different texture and uneven surface for 5-10 minutes

\begin{tabular}{|c|c|c|c|c|c|c|c|c|}
\hline & \multicolumn{2}{|c|}{ Right side } & \multicolumn{2}{|c|}{ Left Side } & \multicolumn{2}{|c|}{ Right side } & \multicolumn{2}{|l|}{ Left side } \\
\hline & Active & Passive & Active & Passive & Active & Passive & Active & Passive \\
\hline \multicolumn{9}{|l|}{ 1.Hip Jt. } \\
\hline \multirow{4}{*}{$\begin{array}{l}\text { Flexion } \\
\text { Extension } \\
\text { Abduction } \\
\text { Adduction }\end{array}$} & $0-85$ & $0-90$ & $0-90$ & $0-95$ & $0-115$ & $0-120$ & $0-115$ & $0-120$ \\
\hline & $0-25$ & $0-30$ & $0-30$ & $0-30$ & $0-30$ & $0-30$ & $0-30$ & $0-30$ \\
\hline & $0-30$ & $0-35$ & $0-40$ & $0-45$ & $0-45$ & $0-45$ & $0-45$ & $0-50$ \\
\hline & $0-15$ & $0-20$ & $0-18$ & $0-20$ & $0-30$ & $0-30$ & $0-30$ & $0-30$ \\
\hline \multicolumn{9}{|l|}{ 2.Knee Jt. } \\
\hline \multirow{2}{*}{$\begin{array}{l}\text { Flexion } \\
\text { Extension }\end{array}$} & $0-110$ & $0-115$ & $0-125$ & $0-130$ & $0-130$ & $0-130$ & $0-125$ & $0-130$ \\
\hline & $110-0$ & $115-0$ & $120-0$ & $130-0$ & $130-0$ & $130-0$ & $120-0$ & $130-0$ \\
\hline \multicolumn{9}{|l|}{ 3. Ankle Jt. } \\
\hline \multirow{2}{*}{$\begin{array}{l}\text { Plantarflexion } \\
\text { Dorsiflexion }\end{array}$} & $0-30$ & $0-35$ & $0-15$ & $0-20$ & $0-40$ & $0-45$ & $0-45$ & $0-45$ \\
\hline & $0-8$ & $0-12$ & NA & $0-10$ & $0-15$ & $0-20$ & $0-20$ & $0-20$ \\
\hline
\end{tabular}

Table 6. Muscle strength

\begin{tabular}{l|c|c|c|c|}
\hline \multirow{2}{*}{\begin{tabular}{l}
\multirow{2}{*}{ Hip } \\
Flexors
\end{tabular}} & \multicolumn{2}{|c|}{ PRE } & \multicolumn{2}{c|}{ POST } \\
\cline { 2 - 5 } & Right side & Left Side & Right side & Left side \\
\cline { 2 - 5 } Extensors & 5 & 5 & 5 & 5 \\
\hline Abductors & 5 & 5 & 5 & 5 \\
\hline Adductors & 5 & 5 & 5 & 5 \\
\hline Knee & 5 & 5 & 5 & 5 \\
\hline Flexors & 5 & 5 & 5 & 5 \\
\hline Extensors & 5 & 5 & 5 & 5 \\
\hline Ankle & 2 & 2 & 4 & 4 \\
\hline Plantarflexors & 2 & 0 & 3 & 2 \\
\hline Dorsiflexors & 2 & 0 & 3 & 3 \\
\hline Toe extensors & 2 & 2 & 4 & 4 \\
\hline Toe flexors & & & & \\
\hline
\end{tabular}

Table 7. Reflexes

\begin{tabular}{|l|c|c|c|c|}
\hline \multirow{2}{*}{} & \multicolumn{2}{|c|}{ PRE } & \multicolumn{2}{c|}{ POST } \\
\cline { 2 - 5 } & Right side & Left side & Right side & Left side \\
\hline \multirow{2}{*}{ Knee (L2, L3, L4) } & $2+$ & $3+$ & $2+$ & $2+$ \\
\hline Ankle (S1, S2) & 0 & 0 & $1+$ & $2+$ \\
\hline Plantar (L5, S1) & Mute & mute & $1+$ & $1+$ \\
\hline
\end{tabular}


Sitting frenkel exercises were started for the patients. The exercises were with specific commands, they were 1) Place foot on therapist's hand, which will change position.2) Raise leg and put foot on traced footprint on the wall. 3) Rise and sit with knees together. Patient did it precisely with 10 repetitions of each activity, twice a day

Coordination Training Coordination exercises were started as a prerequisite for gait training and in order to prevent fall and maintain stability. Tests were used in the form of exercise, they were tapping (foot), alternate heel to knee and knee to toe, drawing a circle with foot, heel on shin. 10 repetitions of each exercise with 3 sets, twice daily was done by the patient.

Along with this, some equilibrium tests for sitting as well as standing were given i.e sitting with support followed by without support progressed to weight shifts in all the direction. Standing exercises were given after patient achieved effective trunk control. They were standing unsupported, eyes closed, with feet together, standing on one foot, tandem standing.

\section{Week12 To Week 20}

Progressive Resisted Exercise were started for patient for effective muscle strengthening. Patients Repetition Maximum (RM) was calculated and strengthening exercises were given 10 repetitions per set, 3 sets twice daily for each major group of muscles. Progression were done gradually.

Progression in the dosage of core strengthening exercise were done with 30 seconds holds, 10 repetitions twice a day.

\begin{tabular}{|c|c|c|c|c|}
\hline & \multicolumn{2}{|c|}{ PRE } & \multicolumn{2}{|c|}{ POST } \\
\hline & Right side & Left side & Right side & Left side \\
\hline \multicolumn{5}{|l|}{ Non equilibrium tests } \\
\hline Tapping(foot) & 3 & 3 & 4 & 4 \\
\hline Alternate heel to knee & 2 & 2 & 4 & 4 \\
\hline Alternate heel to toe & 2 & 3 & 4 & 4 \\
\hline Heel on shin & 1 & 2 & 3 & 3 \\
\hline Drawing a circle(foot) & 3 & 3 & 4 & 4 \\
\hline
\end{tabular}

\begin{tabular}{|l|c|c|}
\hline & Pre treatment & Post treatment \\
\hline Equilibrium tests & & \\
\hline SITTING & & \\
\hline -in normal comfortable position & Good & Good \\
\hline -weight shift in all directions & Fair & Good \\
\hline -multidirectional functional reach & Fair & Good \\
\hline STANDING & & Good \\
\hline -on one foot & Poor & Fair \\
\hline -tandem standing & Poor & Fair \\
\hline -Rhombergs (eyes open to eyes close) & Absent & Fair (with eyes open) \\
\hline -Sharpened Rhombergs (E0 to EC) & Absent & poor(with eyes close) \\
\hline WALKING & & Food \\
\hline -March in place & Absent & Fair \\
\hline -Sideways walk & Absent & Poor \\
\hline -backward walk & Absent & \\
\hline -obstacle walking & & \\
\hline
\end{tabular}

Standing and walking frenkel exercises were added along with sitting exercises with 10 repetitions for each activity. They were- 1 . Place foot forward and backward on a straight line. 2. Walk along a winding strip. 3. Walk between two parallel lines. 4. Walk, placing each foot in a tracing on floor.

Balance Training: After achieving effective trunk and leg control, equilibrium exercises for walking i.e spot marching, obstacle walking, walking sideways, backward, forward, walking on toes and on heel were added along with sitting and standing with dosage of 10 repetitions of each exercises, 2 sets, twice daily. Patient tolerance and fatigue level were taken into consideration when exercises were prescribed.

Follow up: All the clinical assessment, diagnostic tool were taken post treatment showed significant progress 
in patients outcome measures

Scales: Toronto Clinical Neuropathy Scoring System was used. It is a quantitative scoring system for evaluating the severity of peripheral neuropathy - primarily for the feet

Pre score- 11/19 which indicates moderate neuropathy Post Score- 4/19 which indicates no neuropathy

B. Foot and Ankle Disability Index-

Pre score- 40/104

Post score- 98/104

Number of Completed Responses (' $n$ '):

$(\max =26)$ Sum of ' $n$ ' Responses:

$=$ sum of $n$ responses $n{ }^{*} 4 \times 100$ FADI Score

C. NPRS- Numeric Pain rating Scale

Pre score- $7 / 10$

Post score- $2 / 10$

\section{DISCUSSION}

We report case of polyneuropathy along with lumbar radiculopathy. Patients main concern was low back pain initially attention was diverted from neuropathy, as supported by literatures, this is something very common(. However facility of electro-diagnosis helps to form the diagnosis and presence of $\mathrm{Cu}$ deficiency as reveal by lab investigation reports further helps in confirmation as copper deficiency is one of the most common cause of polyneuropathy (Kumar, 2006; Copper, 2020 Strength, in this case was that the patient had a strong family support and she performed all the given interventions perfectly in the given amount of time. Patient were suffering from forgetfulness, behavior problems, poor comprehension which interfered with the intervention process and she had financial instability due to which she was unable to pay medical expenses for every diagnostic as well as intervention process. The treatment initiated with application of deep heating modalities as it is beneficial in order to increase tissue extensibility as well as to increased efficacy of stretching techniques (Taylor, 1995) Lumbar corset were advised to patient.

It shows significant decrement of pain score in daily activities in comparison with patients who did not wear the corset. Also, in combination with exercises, it helps to reduce the radiological instability (Kalichman et al, 2008) Ankle-Foot-Orthosis (AFO) were advised to patient and was used regularly in conjunction with exercise as it reduces the risk of falling besides enhancing mobility (Everaert et al, 2013) While applying stretching technique, proper duration and intensity is extremely significant. 15- 30 seconds of stretching was found to be of the most effective duration since the duration of applications which last longer than 30 seconds did not show any further increase in extensibility (Reddy et al., 2016).
Electrical stimulation were used in the early phase of rehabilitation as slowly increasing triangular pulse is required to stimulate a particular denervated muscle, because the muscle has less capacity to accommodate. So as to achieve a contraction in a denervated muscle, triangular pulses with longer duration is the best choice of treatment(Pieber et al, 2015). Effective core strengthening exercises were given as patient suffered from degenerative spine. There is enough evidence which supports that specific exercise treatment program involving the specific training of the deep abdominal muscles as well as multifidus activation exercises showed a statistically significant reduction in pain intensity and functional disability level.

An important characteristic of sensory ataxia is losses of vibratory sense in the extremities as well as deep tendon reflexes (6). So, sensory retraining were given along with application of vibratory massager in order to overcome inability of vibratory senses. Frenkel exercises were given to patient. It shows significant improvement in lower extremity ataxia patient as it provides fixation by establishing balance between the eccentric and concentric contractions in the multi-joint movements of the lower extremities( Ojoga et al., 2013).

Decreased sensory function of foot and ankle is associated with decreased performance of static and dynamic postural stability. To maintain upright posture, it is necessary to achieve effective trunk and lower extremity control (Van et al, 1999). Hence, patient underwent co-ordination training as well as balance training.Balance training were gradually progressed from static components to dynamic components of balance. Static stabilization were strengthened through external perturbation. Afterwards, the patient were trained in these positions for weight transferring so as to be prepared for dynamic stabilization. Then, progressions were made in which the support surface is narrowed or the center of gravity is changed in order to make the balance activities difficult. Few of the related articles were reported (Jain, et al; Rathi, 2019; Regmi et al., 2019). Khanam et. al. conducted assessment of work-related musculoskeletal morbidity among brick field workers (Khanam et al, 2015).

\section{CONCLUSION}

The case study concluded that there is a significant effect of the given therapeutic intervention on the muscle strength, muscle re-education, co-ordination, balance, gait and ADLs of the patient after 20 weeks of intensive physiotherapeutic intervention.

\section{REFERENCES}

Allen JA. 2020 The Misdiagnosis of CIDP: A Review. Neurol Ther. 26;9(1):43-54.

Berry JA, Elia C, Saini HS, Miulli DE. A Review of Lumbar Radiculopathy, Diagnosis, and Treatment. Cureus 2020: https://www.ncbi.nlm.nih.gov/pmc/ articles/PMC6858271/ 
BF, Waring CA, Brashear TA 1995. The Effects of Therapeutic Application of Heat or Cold Followed by Static Stretch on Hamstring Muscle Length. J Orthop Sports Phys Ther. 21(5):283-6.

Clinical and Diagnostic Findings in Patients with Lumbar ... / clinical-and-diagnostic-findings-in-patients-withlumbar.pdf / PDF4PRO [Internet]. PDF4PRO. 2019 [cited 2020 Dec 6]. Available from: https://pdf4pro.com/view/ clinical-and-diagnostic-findings-in-patients-withlumbar-5af6b8.html

Clinical Orthopaedic Rehabilitation: An Evidence-Based Approach - 3rd Edition 2020 https://www.elsevier.com/ books/clinical-orthopaedic-rehabilitation-an-evidencebased-approach/brotzman/978-0-323-05590-1

Copper deficiency myelopathy produces a clinical picture like subacute combined degeneration | Neurology 2020 https://n.neurology.org/content/63/1/33.short

Della-Giustina D 2015, Evaluation and treatment of acute back pain in the emergency department. Emerg Med Clin North Am, 3(2):311-26.

Dyck PJ, Oviatt KF, Lambert EH. 1981, Intensive evaluation of referred unclassified neuropathies yields improved diagnosis. Ann Neurol.10(3):222-6.

Everaert DG, Stein RB, Abrams GM, Dromerick AW, Francisco GE, Hafner BJ, et al. 2013 Effect of a Foot-Drop Stimulator and Ankle-Foot Orthosis on Walking Performance After Stroke: A Multicenter Randomized Controlled Trial. Neurorehabil Neural Repair. 1;27(7):57991.

Jain, J., S. Banait, I. Tiewsoh, and M. Choudhari. 2018 Kikuchi's Disease (Histiocytic Necrotizing Lymphadenitis): A Rare Presentation with Acute Kidney Injury, Peripheral Neuropathy, and Aseptic Meningitis with Cutaneous Involvement." Indian Journal of Pathology and Microbiology 61(1); 113-15. https://doi. org/10.4103/IJPM.IJPM_256_17.

James, Spencer L, Chris D Castle, Zachary V Dingels, Jack T Fox, Erin B Hamilton, Zichen Liu, Nicholas L S Roberts, et al. "Global Injury Morbidity and Mortality from 1990 to 2017: Results from the Global Burden of Disease Study 2017.” Injury Prevention 26, no. Supp 1 (October2020): i96-114. https://doi.org/10.1136/ injuryprev-2019-043494.

James, Spencer L, Chris D Castle, Zachary V Dingels, Jack T Fox, Erin B Hamilton, Zichen Liu, Nicholas L S Roberts, et al. 2020 Estimating Global Injuries Morbidity and Mortality: Methods and Data Used in the Global Burden of Disease 2017 Study." Injury Prevention 26, i125-53. https://doi.org/10.1136/injuryprev-2019043531 .

Kalichman L, Hunter DJ. 2008 Diagnosis and conservative management of degenerative lumbar spondylolisthesis. Eur Spine J. 17(3):327-35.

Khanam, N., V. Wagh, A.M. Gaidhane, and S.Z. Quazi. 2019 Assessment of Work-Related Musculoskeletal Morbidity, Perceived Causes and Preventive Activities Practiced to Reduce Morbidity among Brick Field
Workers.” Indian Journal of Community Health 31(2) 213-19.

Kumar N 2006. Copper Deficiency Myelopathy (Human Swayback). Mayo Clin Proc. 1;81(10):1371-84.

Latchoumi, T.P., Ezhilarasi, T.P. and Balamurugan, K., 2019. Bio-inspired weighed quantum particle swarm optimization and smooth support vector machine ensembles for identification of abnormalities in medical data. SN Applied Sciences, 1(10), pp.1-10.

Martyn CN, Hughes RA 1997, Epidemiology of peripheral neuropathy. J Neurol Neurosurg Psychiatry. 62(4):310-8.

Ojoga F, Marinescu S. 2013 Physical Therapy and Rehabilitation for Ataxic Patients. Balneo Res J. 20;4(2):81-4.

Peripheral neuropathy - The Lancet [Internet]. 2020. Available from: https://www.thelancet.com/journals/ lancet/article/PIIS0140-6736(04)16508-2/fulltext

Peroneal neuropathy misdiagnosed as L5 radiculopathy: a case report | Chiropractic \& Manual Therapies 2020. https://chiromt.biomedcentral.com/ articles/10.1186/2045-709X-21-12

Physical Medicine and Rehabilitation - 4th Edition 2020. Available from: https://www.elsevier.com/books/ physical-medicine-and-rehabilitation/braddom/978-14377-0884-4

Pieber K, Herceg M, Paternostro-Sluga T, Schuhfried 0 2015. Optimizing stimulation parameters in functional electrical stimulation of denervated muscles: a cross-sectional study. J NeuroEngineering Rehabil. 7;12(1):51.

Rathi, N., B. Taksande, and S. Kumar (2019). "Nerve Conduction Studies of Peripheral Motor and Sensory Nerves in the Subjects with Prediabetes." Journal of Endocrinology and Metabolism 9(5): 147-50. https:// doi.org/10.14740/jem602.

Reddy RS, Alahmari KA 2016. Effect of Lower Extremity Stretching Exercises on Balance in Geriatric Population. Int J Health Sci. 10(3):389-95.

Regmi PR, van Teijlingen E, Mahato P, Aryal N, Jadhav N, Simkhada P, et al. 2019, The health of Nepali migrants in India: A qualitative study of lifestyles and risks. International Journal of Environmental Research and Public Health, 16(19). https://doi.org/10.3390/ ijerph16193655.

Singh R, Sarkar A, Rizvi (Syed) Mohammed, Rasheed M. A 2015. case of peripheral neuropathy due to hypothyroidism misdiagnosed as lumbar radiculopathy: A case report. Indian J Pain. 1;29:181.

Van Deursen RWM, Simoneau GG. 1999 Foot and Ankle Sensory Neuropathy, Proprioception, and Postural Stability. J Orthop Sports Phys Ther. 29(12):718-26.

Zhang X, Zhang Z, Wen J, Lu J, Sun Y, Sang D. 2018 The effectiveness of therapeutic strategies for patients with radiculopathy: A network meta-analysis. Mol Pain https://www.ncbi.nlm.nih.gov/pmc/articles/ PMC5900815/ 